This item was submitted to Loughborough's Research Repository by the author.

Items in Figshare are protected by copyright, with all rights reserved, unless otherwise indicated.

\title{
Nonanalytic behavior of two-dimensional itinerant ferromagnets
}

PLEASE CITE THE PUBLISHED VERSION

http://dx.doi.org/10.1103/PhysRevB.77.220401

\section{PUBLISHER}

(c) The American Physical Society

VERSION

VoR (Version of Record)

LICENCE

CC BY-NC-ND 4.0

REPOSITORY RECORD

Efremov, Dmitry V., Joseph J. Betouras, and Andrey Chubukov. 2019. "Nonanalytic Behavior of Twodimensional Itinerant Ferromagnets". figshare. https://hdl.handle.net/2134/12751. 


\title{
Nonanalytic behavior of two-dimensional itinerant ferromagnets
}

\author{
Dmitry V. Efremov, ${ }^{1}$ Joseph J. Betouras, ${ }^{2}$ and Andrey Chubukov ${ }^{3}$ \\ ${ }^{1}$ Institute for Theoretical Physics, Technical University of Dresden, 01062 Dresden, Germany \\ ${ }^{2}$ Scottish Universities Physics Alliance, Department of Physics and Astronomy, University of St. Andrews, \\ St. Andrews KY16 9SS, Scotland, United Kingdom \\ ${ }^{3}$ Department of Physics, University of Wisconsin-Madison, 1150 University Avenue, Madison, Wisconsin 53706, USA
}

(Received 9 May 2008; published 9 June 2008)

\begin{abstract}
We consider an ordered ferromagnet in the vicinity of a $T=0$ transition into a paramagnet. We show that the free energy and the transverse and longitudinal static susceptibilities contain nonanalyticities which destroy a continuous second-order transition. Depending on the parameters, the transition either becomes first order or occurs via an intermediate spiral phase.
\end{abstract}

DOI: 10.1103/PhysRevB.77.220401

\section{INTRODUCTION}

In recent years, there has been a strong interest to understand hidden features of a $T=0$ ferromagnetic transition in itinerant fermionic systems. A much studied Hertz-Millis-Moriya ${ }^{1-3} \phi^{4}$ model of a ferromagnetic quantum criticality predicts that the transition should be continuous in all dimensions $D>1$, with mean-field exponents, like a classical transition in $D>4=1+z$, where $z=3$ is the dynamical exponent. Meanwhile, experimentally, ${ }^{4}$ pressuretuned ferromagnetic transition in itinerant ferromagnets either becomes first order at low $T$, as in $Z r Z n_{2}$ or $F e G e$, or involves spiral spin ordering, as in $\mathrm{MnSi}$.

Recent theoretical studies ${ }^{5-8}$ revealed that the difference between quantum and classical cases is more than the change of the effective dimension-in the quantum case, the $\phi^{4}$ and higher-order terms in the expansion in the order parameter field contain singular dynamic prefactors which reflect the fact that fermions give rise to long-range, dynamical interaction between collective spin excitations. These dynamic terms have been analyzed on the paramagnetic side of the transition, both in $D=3$ (Ref. 6) and $D=2$ (Ref. 9), and have been found to give rise to two competing effects: (i) the expansion of the free energy in the magnetic field is nonanalytic, and the nonanalytic term favors a preemptive firstorder transition to a state with a finite magnetization, (ii) the static spin susceptibility $\chi(q)$ is nonanalytic in $q$ and becomes negative at some $q_{0}$, signaling another preemptive instability, this time toward a spiral phase. Which instability of a paramagnet comes first depends on the interplay between the prefactors for the analytic $\Delta^{4}$ and $q^{2}$ terms, but in any event, a continuous second-order ferromagnetic transition point is internally unstable.

In this Rapid Communication, we consider what happens when the system approaches a ferromagnetic quantumcritical point (QCP) from the ordered state. We show that the free energy of a quantum ferromagnet is nonanalytic in the order parameter field $\Delta$, and the nonanalytic term favors a first-order transition into a paramagnet at some $\Delta_{c}$. We analyze the static spin susceptibility, which in the ferromagnetic phase has nonequal longitudinal and transverse components, $\chi_{\|}(q)$ and $\chi_{\perp}(q)$, and show that both are nonanalytic in momentum $q$ and both become negative at a finite $q$ inside the ferromagnetic case, when $\Delta$ becomes smaller than some critical $\Delta_{c \perp}$ and $\Delta_{c \|}$. The negative $\chi_{\|, \perp}(q)$ implies the devel-
PACS number(s): 75.40.Cx, 64.70.Tg, 71.10.Ay, 71.10.Hf

opment of the spiral order, either along the direction of the magnetization, or in a transverse direction. We argue that $\Delta_{c}, \Delta_{c \perp}$ and $\Delta_{c \|}$ all scale with the Fermi energy $\varepsilon_{F}$ and depend on a single dimensionless parameter of the model. If $\Delta_{c}>\Delta_{c \perp}, \Delta_{c \|}$, the transition is first order, otherwise, the ferromagnetic phase first becomes unstable against a spiral.

Our analysis is based on the Eliashberg-type consideration near a ferromagnetic QCP. Such approximation has been justified in Refs. 7-9 and we assume that it is valid. For definiteness, we only consider 2D case, but we verified that the conclusions are valid also for 3D systems.

The point of departure of our analysis is the spin-fermion model near a QCP. It describes fermions interacting with their own collective excitations in the spin channel, described by spin variables $\mathbf{S}=c_{\alpha}^{\dagger} \sigma_{\alpha \beta} c_{\beta}$. The model does not assume a long-range order a priori and is described by the Hamiltonian with three terms: $H_{f}=\Sigma_{k, \alpha=\uparrow, \downarrow} \varepsilon_{\mathbf{k}} c_{\mathbf{k}, \alpha}^{\dagger} c_{\mathbf{k}, \alpha}$, which describes low-energy fermions with the dispersion $\varepsilon_{\mathbf{k}} \approx \varepsilon_{F}+v_{F}\left(k-k_{F}\right), \quad H_{s}=\Sigma_{q} \chi_{0}^{-1}(q) \mathbf{S}_{\mathbf{q}} \mathbf{S}_{-\mathbf{q}}$, which describes collective bosonic excitations with a bare static propagator $\chi_{0}(q)$, and the spin-fermion interaction term $H_{\text {int }}$ $=g(1 / N) \Sigma_{\mathbf{k}, \mathbf{q}, \alpha, \alpha^{\prime}} c_{\mathbf{k}, \alpha}^{\dagger} \sigma_{\alpha, \alpha^{\prime}} \mathbf{S}_{\mathbf{q}} c_{\mathbf{k}+\mathbf{q}, \alpha^{\prime}}$. Within this model, $\chi\left(q, \Omega_{m}\right)=\chi_{0}(q) /\left[1-2 g^{2} \chi_{0}(q) \Pi\left(q, \Omega_{m}\right)\right]$, where $\chi_{0}(q)$ is the static susceptibility of free fermions, and $\Pi\left(q, \Omega_{m}\right)$ is the polarization operator (here and below we set the Bohr magneton $\left.\mu_{B}=1\right)$. Near a ferromagnetic transition, $\chi(q)=2 \nu\left(\varepsilon_{F}\right) /\left[\delta+(a q)^{2}\right]$, where $\nu\left(\varepsilon_{F}\right)$ is the density of states per particle at the Fermi surface $\left[\nu\left(\varepsilon_{F}\right)=m /(2 \pi)\right.$ for $\left.\varepsilon_{0}(k)=k^{2} /(2 m)\right], \delta=1-\left[2 g \nu\left(\varepsilon_{F}\right)\right]^{2}$, and the length $a$ is proportional to the radius of the interaction. ${ }^{9}$ The dynamic fermionic self-energy and the dynamic part of $\Pi\left(q, \Omega_{m}\right)$ are computed self-consistently within the model. The fully renormalized spin susceptibility in the paramagnetic phase is

$$
\chi_{i j}\left(q, \Omega_{m}\right)=\delta_{i j} \frac{2 \nu\left(\varepsilon_{F}\right)}{\delta+(a q)^{2}+\bar{\Pi}\left(q, \Omega_{m}\right)},
$$

where $\bar{\Pi}\left(q, \Omega_{m}\right)=\Pi\left(q, \Omega_{m}\right)-\Pi(q, 0)$. For free fermions, $\bar{\Pi}\left(q, \Omega_{m}\right)=\left|\Omega_{m}\right| / \sqrt{\left(v_{F} q\right)^{2}+\Omega_{m}^{2}}$.

The ferromagnetic transition occurs when $g=1 /\left[2 \nu\left(\varepsilon_{F}\right)\right]$. At larger $g, \delta$ becomes negative, and the system develops a ferromagnetic long-range order with the magnetization $M=\left\langle S^{z}\right\rangle=\left(N_{\uparrow}-N_{\downarrow}\right)$. We assume that such long-range order 
does exist and search for preemptive instabilities upon approaching QCP from the ferromagnetic side.

\section{MEAN-FIELD ANALYSIS}

At the mean-field level, the interaction term reduces to $g\left\langle S_{z}\right\rangle \sum_{k, \alpha} \operatorname{sign} \alpha c_{\mathbf{k}, \alpha}^{\dagger} c_{\mathbf{k}, \alpha}$, and the fermionic propagator becomes

$$
G_{\uparrow, \downarrow}^{-1}\left(k, \omega_{m}\right)=i \omega_{m}-\left(\varepsilon_{k}-\mu\right) \pm \Delta_{0},
$$

where $\Delta_{0}=g\left\langle S_{z}\right\rangle=g\left(N_{\uparrow}-N_{\downarrow}\right)$, and $\mu=\mu\left(\Delta_{0}\right)$ is the exact chemical potential (to order $\Delta_{0}^{2}, \mu\left(\Delta_{0}\right)=\mu(0)-\nu^{\prime} /(2 \nu) \Delta_{0}^{2}$, where $\nu^{\prime}$ is the derivative of the density of states at the Fermi surface). Because $\Delta_{0}$ is finite, the longitudinal and transverse spin propagators become unequal already in the static limit. We have

$$
\begin{aligned}
& \chi^{z z}(q)=\frac{\chi_{0}^{z z}}{1-\left(g \chi_{0}^{z z}\right)^{2}+(a q)^{2}}, \\
& \chi^{x x}(q)=\frac{\chi_{0}^{x x}}{1-\left(g \chi_{0}^{x x}\right)^{2}+(a q)^{2}},
\end{aligned}
$$

where

$$
\begin{gathered}
\chi_{0}^{z z}=-\int \frac{d \omega_{m}}{2 \pi} \int \nu(\varepsilon) d \varepsilon\left[G_{\uparrow}^{2}\left(\varepsilon, \omega_{m}\right)+G_{\downarrow}^{2}\left(\varepsilon, \omega_{m}\right)\right], \\
\chi_{0}^{x x}=-2 \int \frac{d \omega_{m}}{2 \pi} \int \nu(\varepsilon) d \varepsilon G_{\uparrow}\left(\varepsilon, \omega_{m}\right) G_{\downarrow}\left(\varepsilon, \omega_{m}\right) .
\end{gathered}
$$

A simple calculation shows that $\chi^{x x}(0)=\left(N_{\uparrow}-N_{\downarrow}\right) / \Delta_{0}=1 / g$, such that $\chi^{x x}(q)$ diverges at $q \rightarrow 0$, in agreement with the Goldstone theorem. For longitudinal susceptibility, $\chi^{z z}(q)=2 \nu\left(\varepsilon_{F}\right) /\left[\delta_{F}+(a q)^{2}\right], \quad$ where $\delta_{F}=-2 \delta=K \Delta_{0}^{2}$, and $K=-(2 / 3) \nu^{\prime \prime} / \nu$. The theory is only valid when $K>0$, otherwise, the transition is first order by trivial reasons. By order of magnitude, $\delta_{F} \sim\left(\Delta_{0} / \varepsilon_{F}\right)^{2}$.

The dynamic terms $\bar{\Pi}^{x x}\left(q, \Omega_{m}\right)$ and $\bar{\Pi}^{z z}\left(q, \Omega_{m}\right)$ also differ at $\Delta_{0} \neq 0$. Evaluating them using fermionic propagators from Eq. (2), we obtain

$$
\begin{gathered}
\bar{\Pi}^{z z}\left(q, \Omega_{m}\right)=\frac{\left|\Omega_{m}\right|}{\sqrt{\left(v_{F} q\right)^{2}+\Omega_{m}^{2}}}, \\
\bar{\Pi}^{x x}\left(q, i \Omega_{m}\right)=\frac{\left|\Omega_{m}\right|}{\sqrt{\left(v_{F} q\right)^{2}+\left(\Omega_{m}+2 i \Delta_{0}\right)^{2}}} .
\end{gathered}
$$

The mean-field dynamic spin susceptibilities in the ferromagnetic phase are then given by

$$
\begin{gathered}
\chi^{z z}\left(q, \Omega_{m}\right)=\frac{2 \nu\left(\varepsilon_{F}\right)}{\delta_{F}+(a q)^{2}+\bar{\Pi}^{z z}\left(q, \Omega_{m}\right)}, \\
\chi^{x x}\left(q, i \Omega_{m}\right)=\frac{2 \nu\left(\varepsilon_{F}\right)}{(a q)^{2}+\bar{\Pi}^{x x}\left(q, \Omega_{m}\right)} .
\end{gathered}
$$

At small $\Omega_{m}$ and $q, \bar{\Pi}^{x x} \approx-i \Omega_{m} / \Delta$. In this limit, $\chi^{x x}$ $\propto\left((a q)^{2}-i \Omega_{m} / \Delta_{0}\right)^{-1}$ describes transverse Goldstone excita- tions with $\Omega=\Delta_{0}(a q)^{2}$, while $\chi^{z z}$ describes overdamped longitudinal excitations.

\section{ELIASHBERG THEORY}

Equations (2)-(6) constitute the mean-field description of the ferromagnetic phase. Within this description, the transition is continuous, i.e., the ferromagnetic phase is stable up to a point where $\Delta_{0}, \delta=0$. The Eliashberg theory goes beyond this approximation-it self-consistently takes into account $\omega$-dependent fermionic self-energy, but neglects $k$-dependent self-energy and vertex corrections. Vertex corrections generally are not small if the interaction involves small momentum transfers and are necessary to satisfy Ward identities related to the conservation laws. However, the analysis of vertex corrections on the paramagnetic side have shown $n^{8,9}$ that they can be rigorously neglected in the calculations of the nonanalytic terms in the free energy and spin susceptibilities, if the interaction is sufficiently long-ranged such that $a k_{F}>1$, which we assume to hold. ${ }^{10}$

The calculations proceed in three steps, like in the paramagnetic phase. First, we obtain self-consistent one-loop expressions for the fermionic self-energy and dynamic spin susceptibilities. Second, we use these one-loop expressions as inputs, obtain the free energy within Eliashberg theory, and show that it is nonanalytic in $\Delta$. Third, using the same inputs, we compute static spin susceptibilities at the two-loop level and find terms which are nonanalytic in momentum. We argue that the nonanalytic terms in the free energy favor a first-order transition, while the nonanalytic terms in the susceptibilities favor an intermediate spiral phase.

\section{FERMIONIC SELF-ENERGY}

The one-loop fermionic self-energy in the ordered phase is given by

$$
\Sigma_{f}\left(\omega_{m}\right)=\left\{\begin{array}{ll}
\lambda\left(\varepsilon_{k}\right) \omega_{m} & \text { for } \omega_{m} \ll \omega_{0} / \lambda^{3} \\
\omega_{0}^{1 / 3} \omega_{m}^{2 / 3} & \text { for } \omega_{m} \gg \omega_{0} / \lambda^{3}
\end{array},\right.
$$

where $\omega_{0}=3 \sqrt{3} \varepsilon_{F} /\left[4\left(a k_{F}\right)^{4}\right], \varepsilon_{F}=v_{F} k_{F} / 2$, and $\lambda\left(\varepsilon_{k}\right)$ depends in a nonsingular way on the ratio of $\varepsilon_{k}$ and $\Delta_{0}$. For $\varepsilon_{k}=\mu$,

$$
\lambda=\frac{1}{4 a k_{F}}\left[\frac{v_{F}}{a \Delta_{0}}+\frac{1}{\sqrt{\delta_{F}+\left(2 a \Delta_{0} / v_{F}\right)^{2}}}\right] .
$$

The self-energy is linear in $\omega_{m}$ at the smallest frequencies, but crosses over to the quantum-critical, $\omega_{m}^{2 / 3}$ behavior at frequencies larger than $\omega_{0} / \lambda^{3} \sim \Delta_{0}^{3} /\left[\varepsilon_{F}^{2}\left(a k_{F}\right)\right]$. Such selfenergy does not destroy the ferromagnetic order and preserves a Fermi surface, but it is larger than $\omega_{m}$ near QCP and has a nonFermi liquid form in between $\omega_{0} / \lambda^{3} \ll \omega_{0}$ and $\omega_{0}$. The nonFermi liquid behavior in the ferromagnetic state has been discussed from a different point of view in Ref. 11.

The one-loop dynamic polarization operators $\bar{\Pi}_{1}^{x x}\left(q, \Omega_{m}\right)$ and $\bar{\Pi}_{1}^{z z}\left(q, \Omega_{m}\right)$, reevaluated with dressed fermions, are given by rather complex expressions. Like in previous studies, ${ }^{8,9}$ we found that, for the calculations of the nonanalytic terms in the free energy and spin susceptibilities, we only need 
terms up to order $1 / q^{3}$ in the $1 / q$ expansion of $\bar{\Pi}\left(q, \Omega_{m}\right)$. Such terms are not affected by vertex corrections. ${ }^{8}$ We obtained

$$
\begin{gathered}
\bar{\Pi}_{1}^{x x}(q, \omega)=\frac{|\omega|}{v_{F} q}\left[1-\frac{\left(\omega+c_{\omega} \Sigma_{f}(\omega)+2 i \Delta_{0}\right)^{2}}{2 v_{F}^{2} q^{2}}\right], \\
\bar{\Pi}_{1}^{z z}(q, \omega)=\frac{|\omega|}{v_{F} q}\left\{1-\frac{\left[\omega+c_{\omega} \Sigma_{f}(\omega)\right]^{2}}{2 v_{F}^{2} q^{2}}\right\},
\end{gathered}
$$

where $c_{\omega}$ interpolates between $c_{\omega=0}=1$ and $c_{\omega} \approx 1.20$ for $\omega_{0} / \lambda^{3}<\omega<\omega_{0}$.

\section{FREE ENERGY}

The free energy per particle for the ferromagnetic spinfermion model in the Eliashberg approximation is given by

$$
\Xi=\Xi_{0}\left(\Delta_{0}\right)+\Xi^{z z}+2 \Xi^{x x},
$$

where $\Xi_{0}(\Delta)=\frac{2 m}{p_{F}^{2}}\left[-(1 / 4) \delta_{F} \Delta^{2}+(K / 8) \Delta^{4}+\ldots\right]$ is analytic in $\Delta$. Minimizing $\stackrel{p F}{\Xi}_{0}$ and expanding around the minimum, we obtain the equilibrium $\Delta=\Delta_{0}=\left(\delta_{F} / K\right)^{1 / 2}$ and reproduce the mean-field expression for the static $\chi^{z z}(q \rightarrow 0)$. Further,

$$
\begin{aligned}
& \Xi^{z z}=\frac{V}{2 N} \int \frac{d \Omega_{m} d^{2} q}{(2 \pi)^{3}} \ln \frac{\chi^{z z}(0,0)}{\chi^{z z}\left(q, \Omega_{m}\right)}, \\
& \Xi^{x x}=\frac{V}{2 N} \int \frac{d \Omega_{m} d^{2} q}{(2 \pi)^{3}} \ln \frac{\chi^{x x}(0,0)}{\chi^{x x}\left(q, \Omega_{m}\right)},
\end{aligned}
$$

where $\chi^{i i}\left(q, \Omega_{m}\right)$ include one-loop polarization operators $\bar{\Pi}_{1}^{i i}\left(q, \Omega_{m}\right)$. Both $\Xi^{z z}$ and $\Xi^{x x}$ contain analytic contributions which renormalize constants in $\Xi_{0}(\Delta)$. These renormalizations are small in $1 /\left(a k_{F}\right)$ (Ref. 9) and we neglect them. In addition, $\Xi^{x x}$ contains the nonanalytic term in $\Delta$, which is our primary interest. Substituting $\chi^{x x}$ with the polarization operator from Eq. (8) into Eq. (9), integrating over momentum, and neglecting regular terms, we obtain at QCP,

$$
\Xi^{x x}=\frac{\sqrt{2}}{2 \pi c^{3 / 2}} \frac{\Delta^{7 / 2}}{\varepsilon_{F}^{2} \omega_{0}^{1 / 2}} Z,
$$

where $Z$ is the universal (cutoff independent) part of the integral

$$
Z=2 \int_{0}^{A} d y \operatorname{Re}\left[\left(y^{2 / 3}-i\right)^{2} \log \left(y^{2 / 3}-i\right)^{2}\right] .
$$

The evaluation of the integral yields $Z=-8 \pi \sqrt{2} / 35 \approx-1.02$. Combining nonanalytic and analytic terms, we obtain in the immediate vicinity of the QCP,

$$
\Xi=\frac{1}{\varepsilon_{F}}\left(-\frac{\delta_{F}}{4} \Delta^{2}+\frac{K}{8} \Delta^{4}-\frac{\Delta^{7 / 2}}{E^{3 / 2}}\right),
$$

where $E=d \varepsilon_{F} /\left(a k_{F}\right)^{4 / 3}$, and $d \approx 3.50$. Apart from a small numerical difference in $d$, this expression coincides with the one obtained in Ref. 9 where QCP was approached from the paramagnetic side. There is, however, an important distinc-

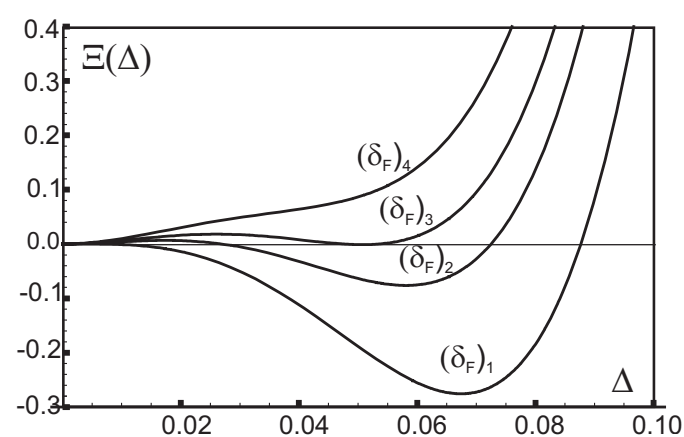

FIG. 1. The free energy $\Xi(\Delta)$, Eq. (12), for different values of $\delta_{F}$, going from positive $\left(\delta_{F}\right)_{1}$ to negative $\left(\delta_{F}\right)_{2,3,4}$. The first-order transition occurs at $\left(\delta_{F}\right)_{3}$.

tion between paramagnetic and ferromagnetic phases away from QCP. In the paramagnetic phase, the $\Delta^{7 / 2}$ dependence is replaced by $\Delta^{3}$ when $\Delta / \varepsilon_{F} \sim \delta /\left(a k_{F}\right)^{2} \leq \delta$. In the ferromagnetic phase, $\delta \rightarrow \delta_{F}$, which by itself scales as $\left(\Delta / \varepsilon_{F}\right)^{2}$. As a result, the nonanalytic $\Delta^{7 / 2}$ dependence of $\Xi$ survives in the wide range away from a QCP, as long as $\Delta / \varepsilon_{F} \leq \gamma$, where $\gamma=\left(a k_{F}\right)^{2} /\left(K \varepsilon_{F}^{2}\right)$.

The negative $\Delta^{7 / 2}$ term in the free energy shifts the equilibrium value $\Delta_{0}$ such that it remains finite even on the paramagnetic side of the transition, when $\delta_{F}$ changes sign and becomes negative. For a generic $\delta_{F}, \Delta_{0}$ is the solution of $K \Delta_{0}^{2}-7\left(\Delta_{0} / E\right)^{3 / 2}=\delta_{F}$. One can easily verify (see Fig. 1) that the free energy [Eq. (12)] describes a first-order transition to a paramagnet at $\delta_{F}=-\left(\Delta_{0} / E\right)^{3 / 2}<0$ (i.e., $2 \delta=-\delta_{F}>0$ ). The value of the equilibrium $\Delta_{0}$ at such a transition is $\Delta_{c}$ $=36 /\left(K^{2} E^{3}\right) \approx 0.84 \gamma^{2} \varepsilon_{F}$. We also note that a prefactor for $\left(\Delta-\Delta_{0}\right)^{2} / 2$ in the free energy changes from $\delta_{F}$ to $\delta_{F}^{\text {eff }}=\delta_{F}$ $+(7 / 4)\left(\Delta_{0} / E\right)^{3 / 2}$, and remains positive for all $\Delta_{0}>\Delta_{c}$. At $\Delta_{0}=\Delta_{c}, \delta_{F}^{\text {eff }}=(3 / 4)\left|\delta_{F}\right|$.

\section{STATIC SPIN SUSCEPTIBILITIES}

We next show that the static spin susceptibilities $\chi^{x x}(q, 0)$ and $\chi^{z z}(q, 0)$ also display nonanalytic behavior, and that these nonanalyticities compete with the one in the free energy and may give rise to preemptive spiral instabilities. The nonanalytic term in $\chi(q, 0)$ has been previously analyzed on the paramagnetic side. ${ }^{8,9}$ We performed the calculations in the ordered phase.

The nonanalytic behavior of $\chi(q, 0)$ originates from nonanalytic $q$-dependencies of $\Pi^{z z}$ and $\Pi^{x x}$, which acquire static parts at the two-loop order.

The computational steps are similar to those in Ref. 8 and we refrain from discussing them. The nonanalytic contributions $\Pi_{2}^{z z}$ and $\Pi_{2}^{x x}$ come from the processes in which fermions and spin fluctuations are vibrating near a fermionic mass shell and are far away from a bosonic mass shell ${ }^{7,8}$ [the same processes lead to fermionic self-energy $\left.\Sigma_{f}\left(\omega_{m}\right)\right]$. We found that the nonanalyticity comes from the exchange processes involving transverse spin fluctuations.

There are two types of nonanalyticities in an ordered ferromagnet. First, there are corrections to static, uniform $\Pi^{z z}(0,0)$. They change $\delta_{F}$ into $\delta_{F}^{\text {eff }}$, which is the same as the 


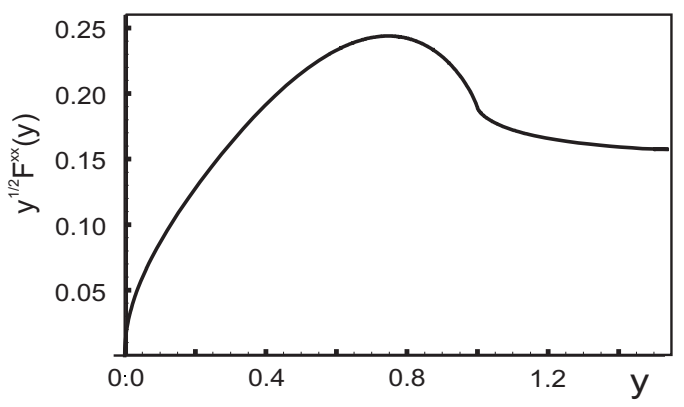

FIG. 2. The scaling function $\sqrt{y} F^{x x}(y)$. It has a maximum value of $\sim 0.24$ at $y \approx 0.74$.

stiffness obtained by expanding the free energy. Second, there are nonanalytic terms in the momentum expansion of the static $\Pi^{z z}(q, 0)$ and $\Pi^{x x}(q, 0)$ Combining both, we found

$$
\begin{gathered}
\chi^{z z}(q, 0)=\frac{2 \nu\left(\varepsilon_{F}\right)}{a^{2}} \frac{1}{\frac{\delta_{F}^{\text {eff }}}{a^{2}}+q^{2}-q^{3 / 2} k_{F}^{1 / 2} F^{z z}\left(\frac{2 \Delta_{0}}{v_{F} q}\right)}, \\
\chi^{x x}(q, 0)=\frac{2 \nu\left(\varepsilon_{F}\right)}{a^{2}} \frac{1}{q^{2}-q^{3 / 2} k_{F}^{1 / 2} F^{x x}\left(\frac{2 \Delta_{0}}{v_{F} q}\right)} .
\end{gathered}
$$

For $\Delta_{0} \ll v_{F} q, F^{z z}(0)=F^{x x}(0) \approx 0.25$, in agreement with Ref. 8 . For $v_{F} q \ll \Delta_{0}$, the two scaling functions differ. The scaling function $F^{z z}(y)$ remains close to $F^{z z}(0)$ as long as the argument $y<\varepsilon_{F} / \Delta_{0}$. The function $F^{x x}(y)$ crosses over to $F^{x x}(y \gg 1) \approx 0.15 / \sqrt{y}$ such that $\Pi_{2}^{x x}(q, 0)$ becomes analytic, $\Pi_{2}^{x x}(q, 0) \propto q^{2}$. The function $\sqrt{y} F^{x x}(y)$ is plotted in Fig. 2. Analyzing Eq. (13), we find that both susceptibilities become negative at a finite $q$ when $\Delta_{0}$ reduces below some critical value. The transverse susceptibility becomes negative at $\Delta_{0}=\Delta_{c, \perp}=\varepsilon_{F}[\sqrt{y} F(y)]_{\max }^{2}=0.06 \varepsilon_{F}$, whereas longitudinal susceptibility becomes negative at $\Delta_{0}=\Delta_{c, \|}$, which is the solution of

$$
\frac{\Delta_{c, \|}}{\varepsilon_{F}}\left(1-\frac{21}{4} \frac{\gamma}{d^{3 / 2}} \sqrt{\frac{\varepsilon_{F}}{\Delta_{c, \|}}}\right)^{1 / 2}=0.02 \sqrt{\gamma} .
$$

Whether the transition is the first order or involves an intermediate spiral phase depends on which of $\Delta_{c}, \Delta_{c, \|}$, and $\Delta_{c, \perp}$ is the largest. All three critical $\Delta$ scale with $\varepsilon_{F}$, but they

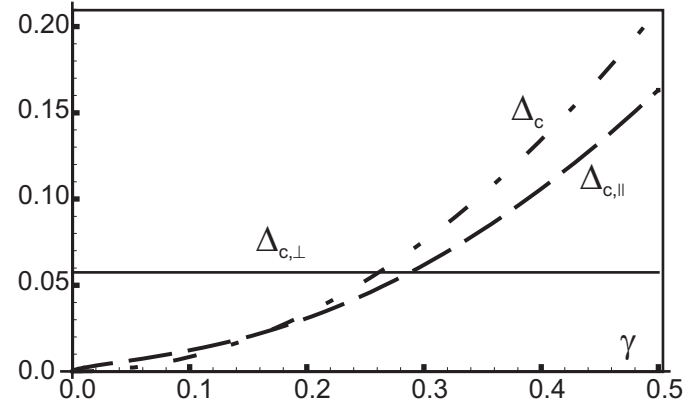

FIG. 3. The three critical $\Delta_{c}$ as functions of the parameter $\gamma=\left(a k_{F}\right)^{2} /\left(K \varepsilon_{F}^{2}\right)$.

depend differently on the parameter $\gamma$. In Fig. 3 we plotted the three critical $\Delta$ vs $\gamma$. We see that for $\gamma<0.26$, $\Delta_{c, \perp}>\Delta_{c, \|}, \Delta_{c}$, and the system first develops a transverse spiral order, while for $\gamma>0.26, \Delta_{c}>\Delta_{c, \|}, \Delta_{c, \perp}$, and the transition is first order.

In summary, we analyzed the nonanalytic terms in the free energy and in static susceptibilities in an ordered itinerant ferromagnet. We found that, because of these nonanalyticities, the transition to a paramagnet is either first order, or involves an intermediate spiral phase. Nonanalyticities in the dispersion of Goldstone modes in itinerant ferromagnets were discussed in Ref. 12. They found in 2D $\Omega \Delta_{0} \propto q^{2} / \Delta_{0}$, i.e., $\Omega \propto q^{2}$, with the prefactor independent on $\Delta_{0}$. Our result differs from theirs-from Eq. (1), we extract $\omega$ $=q^{2} \Delta_{0}\left(1-\left(\Delta_{c, \perp} / \Delta_{0}\right)^{1 / 2}\right)$. This is due to different sign of the nonanalytic term in our case, and its different dependence on $\Delta_{0}$ because of fermionic self-energy.

\section{ACKNOWLEDGMENTS}

We thank A. Andreev, P. Fulde, A. Green, A. Huxley, D. Maslov, A. Rosch, and Yu. Ovchinnikov for useful discussions. The research was supported by NSF Grant No. DMR 0604406 (A.C.) and in part by NSF Grant No. PHY05-51164 (J.B.). J.B. would like to thank KITP, Santa Barbara for hospitality during the completion of this work.
${ }^{1}$ J. Hertz, Phys. Rev. B 14, 1165 (1976).

${ }^{2}$ T. Moriya, Spin Fluctuations in Itinerant Electron Magnetism (Springer-Verlag, Berlin, 1985).

${ }^{3}$ A. J. Millis, Phys. Rev. B 48, 7183 (1993).

${ }^{4}$ N. Dorion-Leyraud et al., Nature (London) 425, 595 (2003);

M. Uhlarz et al., Phys. Rev. Lett. 93, 256404 (2004);

P. Pedrazzini et al., ibid. 98, 047204 (2007).

${ }^{5}$ D. Belitz, T. R. Kirkpatrick, and T. Vojta, Rev. Mod. Phys. 77, 579 (2005).

${ }^{6}$ D. Belitz, T. R. Kirkpatrick, and T. Vojta, Phys. Rev. B 55, 9452 (1997).

${ }^{7}$ A. V. Chubukov, C. Pepin, and J. Rech, Phys. Rev. Lett. 92, 147003 (2004).
${ }^{8}$ J. Rech, C. Pepin, and A. V. Chubukov, Phys. Rev. B 74, 195126 (2006).

${ }^{9}$ D. L. Maslov, A. V. Chubukov, and R. Saha, Phys. Rev. B 74, 220402(R) (2006).

${ }^{10} \mathrm{An}$ alternative way to justify Eliashberg theory even if $a k_{F} \leq 1$ is to extend the model to $M \gg 1$ species of fermions, which separately interact with a collective boson. Vertex corrections are then small in $1 / M$.

${ }^{11}$ V. P. Mineev, Phys. Rev. B 72, 144418 (2005); D. Belitz, T. R. Kirkpatrick, and A. Rosch, ibid. 74, 024409 (2006).

${ }^{12}$ D. Belitz, T. R. Kirkparick, A. J. Millis, and T. Vojta, Phys. Rev. B 58, 14155(R) (1998). 